\title{
Tendencia del NDVI en el período 2000-2014 como indicador de la degradación de tierras en Argentina: ventajas y limitaciones
}

Gaitán, J.J.; D.E. Donaldo Bran y C. Azcona

\begin{abstract}
RESUMEN
La degradación de tierras es uno de los mayores problemas ambientales de la Argentina. Para gestionar esta problemática se requieren sistemas de monitoreo que permitan detectar su tendencia y proporcionen alertas tempranas. Recientemente se han desarrollado metodologías de monitoreo basadas en índices de imágenes satelitales, siendo NDVI (Normalized Difference Vegetation Index) el más utilizado. Se analizó la tendencia del NDVI (del sensor MODIS), entre 2000-2014, como posible indicador de la degradación de tierras en la Argentina. Para cada año (del 01/07 al 30/06 del año siguiente) se calculó la integral anual del NDVI y su tendencia mediante regresión lineal simple para cada pixel. De acuerdo al signo y significancia estadística de la pendiente se cartografiaron áreas con tendencias negativas (TN; 37,9\% del territorio nacional), positivas (TP; $5,0 \%$ ) y sin tendencia $(57,1 \%)$. Las TN se concentraron en Patagonia Norte (asociado a la deposición de cenizas volcánicas y a un ciclo seco) y en el Chaco Seco (asociado a desmontes para agricultura y a un ciclo seco). Las TP se concentraron en el noroeste del país (posiblemente asociado a un ciclo húmedo). Esta metodología, junto con indicadores de campo y datos climáticos, puede integrar un sistema para monitorear las tierras a escala nacional.
\end{abstract}

Palabras clave indicadores, sensores remotos, servicios ecosistémicos

Gaitán, J.J.; D.E. Donaldo Bran and C. Azcona, 2015. Trend of NDVI in the period 2000-2014 as indicator of land degradation in Argentina: advantages and limitations. Agriscientia 32 (2): 83-93

\section{RESUMEN}

Land degradation is one of the main environmental concerns in Argentina. Monitoring systems to detect trend and provide early warnings are required to deal with this issue. Recently, there have been developed monitoring methodologies based on indices derived from satellite images, among them the most widely used is the NDVI (Normalized Difference Vegetation Index). The 
trend of NDVI (from MODIS sensor) was analyzed, between 2000-2014, as a possible indicator of land degradation in Argentina. For each year (from 01-July until 30-June of the following year) the annual integral of NDVI and its trend was calculated using simple linear regression for each pixel. According to the sign and statistical significance of the regression slope were mapped areas with negative trends (TN, 37.9\% of the country), positive (TP, $5.0 \%$ ) and no trend (57.1\%). The TN was concentrated in North Patagonia (associated with the deposition of volcanic ash and a dry cycle) and the Chaco Seco (associated with forest clearing for agriculture and a dry cycle). The TP was concentrated in the northwest of the country (possibly associated with the occurrence of a wet cycle). This methodology, along with other indicators based on field information and climate data, may be useful for developing a monitoring system of land degradation at a national scale.

Key words: indicators, remote sensing, ecosystem services

J.J. Gaitán: Instituto Nacional de Tecnología Agropecuaria (INTA), Instituto de Suelos-CNIA, Argentina. D.E. Donaldo Bran: INTA, EEA Bariloche, Argentina. C. Azcona: Universidad Nacional de Río Negro, Sede Andina, Argentina. Correspondencia a: gaitan.juan@inta.gob.ar

\section{INTRODUCCIÓN}

El problema de la desertificación y degradación de las tierras es generalizado a escala global y ha sido señalado por la Organización de las Naciones Unidas (ONU) como uno de los "aspectos del cambio global más importantes a los que se enfrenta la humanidad" (UNCCD, 1994). En este sentido, dado el reconocimiento de la importancia que esta problemática conlleva a escala mundial, al igual de lo que ocurre con el cambio climático global y la biodiversidad, la ONU establece a partir de 1994 la Convención para la Lucha contra la Desertificación (UNCCD). La República Argentina no es ajena a esta problemática, donde una parte significativa del territorio está sujeto a prácticas ganaderas y agrícolas inapropiadas, con un manejo inadecuado de sus recursos naturales, lo que implica la pérdida de la biodiversidad, de los bosques y del suelo y una progresiva disminución de la productividad, con la consiguiente disminución de la calidad de vida de la población rural (SADyS, 2001).

Pese a los esfuerzos de la UNCCD y de ser un problema ambiental de primera magnitud científica, política y socioeconómica, la desertificación y degradación de tierras provoca desacuerdo y controversia entre científicos, políticos y gestores del territorio. El Millennium Ecosystem Assessment (MEA, 2005) resalta la falta de evaluación y monitoreo de la desertificación, y postula que sin una línea de base científicamente robusta y consistente, la identificación de prioridades y el monitoreo de los resultados de las acciones se ven seriamente restringidos. El establecimiento de programas de seguimiento de los cambios en la cobertura del suelo a largo plazo es una manera efectiva de evaluar el estado de los recursos naturales y la evolución de los procesos de desertificación. Estos programas son una herramienta para detectar cambios tempranos en la estructura y funcionamiento del ecosistema que indiquen la presencia de desertificación, lo que permitiría establecer medidas de gestión efectivas y económicamente viables. Sin embargo, la falta de una metodología que permita estimar la extensión, intensidad y evolución de la desertificación de manera objetiva (Reynolds \& Stafford Smith, 2002), continúa generando escepticismo en la sociedad.

En los últimos años se han desarrollado metodologías para el monitoreo de las tierras basadas en el uso de índices de vegetación derivados de sensores remotos, como estimadores de atributos estructurales y funcionales de los ecosistemas. Entre estos índices, el Normalized Difference Vegetation Index (NDVI) (Tucker, 1979) es uno de los más ampliamente utilizados dado que en diversos estudios se han determinado relaciones significativas entre este índice y aspectos claves de la estructura y funcionalidad de los ecosistemas, como el contenido de biomasa (Tucker et al., 1985), el índice de área foliar (Steltzer \& Welker, 2006), la productividad primaria neta aérea (Paruelo et al., 
1997) y la cobertura vegetal (Gaitán et al., 2013).

La Argentina ha desarrollado un Observatorio Nacional de la Degradación de las Tierras y Desertificación (www.desertificacion.gob.ar), cuya finalidad es cuantificar la naturaleza y la severidad de los impactos de la desertificación/degradación de las tierras y construir capacidades de intervención que permitan atenuar dichos impactos. En el marco de este Observatorio Nacional de la Degradación de las Tierras y Desertificación se realizó el presente estudio cuyo objetivo fue analizar la tendencia temporal del índice NDVI, en el período 2000-2014, como un indicador para monitorear la degradación de las tierras en la República Argentina.

\section{MATERIALES Y MÉTODOS}

Se utilizaron datos de NDVI del producto MOD13Q1 derivados del sensor Moderate Resolution Imaging Spectroradiometer (MODIS), que orbita a bordo del satélite TERRA. El satélite TERRA recorre la superficie terrestre diariamente y registra la reflexión de la Tierra en 36 porciones del espectro electromagnético. Para el cálculo del NDVI se utilizan las bandas correspondientes a la porción del rojo (IR, 620-670 nm) y del infrarrojo cercano (IRc, 841-876 nm) del espectro electromagnético, de acuerdo a la siguiente relación:

$$
\mathrm{NDVI}=(\mathrm{IR}-\mathrm{IRC}) /(\mathrm{IR}+\mathrm{IRC})
$$

Las imágenes del producto MOD13Q1 tienen una resolución espacial de 250 metros (tamaño de pixel: 6,25 hectáreas) y comprenden el período desde febrero de 2000 hasta la actualidad con una resolución temporal de 16 días (23 imágenes por año). Los datos de estas imágenes son compuestos de máximo valor, formados por los mayores valores diarios de cada pixel durante el período de 16 días, están geométrica y atmosféricamente corregidas e incluyen un índice de calidad del dato (pixel reliability), basado en las condiciones ambientales en las cuales se registró el dato (Justice et al., 2002): 0 = confiable (no hay presencia de nubes, nieve, ni aerosoles atmosféricos); 1 = semiconfiable (no hay presencia de nubes ni nieve, el objetivo es visible y las correcciones atmosféricas han mejorado la calidad del dato original); 2 = nieve (superficie cubierta total o parcialmente por nieve); 3 = nubes (superficie total o parcialmente cubierta por nubes); 4 = sin dato (dato ausente por múltiples razones).

Para abarcar todo el territorio de la República Argentina fue necesario obtener nueve imágenes, para cada fecha, de los siguientes path/row: h11v11, h12v11, h12v12, h12v13, h13v11, h13v12, h13v13, h13v14 y h14v14. En total se procesaron 2898 imágenes (9 imágenes $\times 23$ fechas/año $\times 14$ años). A partir de las nueve imágenes de cada fecha se realizó un mosaico para obtener una imagen por fecha que abarca todo el país (23 mosaicos/año $\times 14$ años $=322$ mosaicos).

Para el análisis de la tendencia temporal se consideró cada año como el período comprendido entre el 1 de julio del año $n$ y el 30 de junio del año $n+1$. Se calculó la integral anual del NDVI (NDVI-I) de cada año como la sumatoria de los productos de los 23 valores de NDVI por la fracción del año que comprende cada mosaico. Los datos con pixel reliability mayores a uno fueron reemplazados por la media del NDVI de la estación de crecimiento. De esta manera se obtuvieron 14 mosaicos (años: 2000-01 a 2013-14) donde cada pixel tiene el valor de NDVI-I. En varios estudios se ha mostrado que NDVI-I está fuertemente relacionado con la productividad primaria neta aérea del ecosistema (Tucker et al., 1983; Prince, 1991; Paruelo et al., 1997) que, a su vez, está estrechamente relacionada con el flujo de energía y el ciclo del carbono y nutrientes (Chase et al., 2000). Por lo tanto, la productividad primaria ha sido considerada como un atributo clave de los ecosistemas dado que integra varios aspectos funcionales de éstos (McNaughton et al., 1989).

Se calculó la tendencia temporal de NDVI-I a nivel de cada pixel a lo largo de los 14 años. Para ello se analizó la regresión lineal entre el tiempo (variable $x$ ) y NDVI-I (variable $y$ ) en cada pixel. A partir del signo de la pendiente y de la significancia estadística del análisis de regresión se elaboró un mapa de las áreas con tendencia significativa negativa (coeficiente de correlación de Pearson < -0,53; que es el valor umbral para 12 grados de libertad = 14 años -2 ; y un nivel de probabilidad $p<0,05$ ), tendencia significativa positiva (coeficiente de correlación $>0,53$ ) y sin tendencia significativa (coeficiente de correlación entre $-0,53$ y 0,53 ) para el período 2000 a 2014. Además de conocer la existencia de tendencias negativas o positivas en cada región, es importante establecer las tasas de disminución o aumento NDVI-I para indicar diferentes velocidades de ocurrencia de procesos de deterioro (tasas más negativas) o recuperación (tasas más positivas). Para ello se calculó la pendiente de la regresión entre los años y NDVI-I, y su valor se expresó como porcentaje del valor inicial de la serie (año 2000-01).

El comportamiento de NDVI-I está influenciado por el clima, principalmente las precipitaciones (Wessels et al., 2007). Debido a la ocurrencia del fenómeno de El Niño-Oscilación del Sur (ENSO en sus siglas en inglés), las precipitaciones, en el 
período analizado, pueden haber mostrado tendencias diferentes entre las regiones del país. El ENSO tiene dos extremos: una fase cálida conocida como "El Niño" y una fase fría conocida como "La Niña". Se han desarrollado varios índices para monitorear la ocurrencia de estas fases, uno de los más utilizados es el MEI (Multivariate ENSO Index) (Wolter \& Timlin, 1993). De acuerdo a dicho índice, en la primera mitad del período analizado en este estudio predominó la ocurrencia de eventos El Niño (63\% del tiempo), mientras que en la segunda mitad predominaron los eventos La Niña (67\% del tiempo). No se contó con suficientes series de datos completas de precipitaciones de estaciones meteorológicas que permitan analizar la ocurrencia de ciclos secos o húmedos en las diferentes regiones del país, lo cual podría ayudar a interpretar las causas de las tendencias de NDVI-I. Por lo tanto se realizó el análisis de la superficie de tres grandes lagunas que son el sumidero de grandes cuencas endorreicas de tres regiones del país: lagunas de Carrilaufquen Chica y Grande (41,15 S y 69,45 O, provincia de Río Negro), laguna de Mar Chiquita (30,65 S y 62,65 O, provincia de Córdoba) y laguna de Los Pozuelos (22,35 S y 66,00 O, provincia de Jujuy). Las oscilaciones de la superficie de estos espejos de agua podrían ser indicadoras de la ocurrencia de ciclos secos o húmedos en estas regiones. Para ello se utilizaron imágenes satelitales de los sensores Landsat 5, 7 y 8 de las siguientes fechas: lagunas de Carrilaufquen (path/ row: 230/89), día juliano 06 del año 2000, 24 de 2001, 27 de 2002, 14 de 2003, 41 de 2004, 27 de 2005, 30 de 2006, 33 de 2007, 22 de 2009, 25 de 2010, 20 de 2011, 42 de 2012, 25 de 2013 y 04 de 2014; laguna de Mar Chiquita (path/row: 228/81), 32 de 2000, 50 de 2001, 45 de 2002, 32 de 2003, 27 de 2004, 45 de 2005, 48 de 2006, 35 de 2007, 54 de 2008, 40 de 2009, 27 de 2010, 46 de 2011, 41 de 2012, 43 de 2013 y 22 de 2014; laguna de Los Pozuelos (path/row: 232/75), 316 de 2000, 310 de 2001, 313 de 2002, 308 de 2003, 311 de 2004, 305 de 2005, 316 de 2006, 327 de 2007, 306 de 2008, 308 de 2009, 311 de 2010, 306 de 2011, 293 de 2012, 319 de 2013 y 290 de 2014.

\section{RESULTADOS}

En el período 2000-2014, NDVI-I mostró una tendencia negativa en $37,9 \%$ del territorio nacional, una tendencia positiva en 5,0\%, mientras que en $57,1 \%$ del territorio no hubo un cambio significativo (Figura 1). Las áreas con tendencias negativas se concentraron en el norte de la Patagonia y en la región del Chaco Seco que, además, son las re- giones que mostraron las tasas más acentuadas de disminución en NDVI-I (Figura 2). Las áreas con tendencias positivas se hallaron principalmente en las regiones Altoandina, Puna y Monte de Sierras y Bolsones (Figura 1).

Las provincias con mayor proporción de áreas con tendencia negativa en NDVI-I fueron: Río Negro $(78,8 \%$ del territorio provincial), Santiago del Estero (75,3\%), Córdoba (61,4\%), Chaco (59,9\%) y Tucumán $(55,1 \%)$. Las provincias con mayor proporción de áreas con tendencia positiva en NDVI-I fueron: San Juan (34\%), Jujuy $(31,4 \%)$, Catamarca $(23,1 \%)$, La Rioja $(15,0 \%)$ y Salta $(9,7 \%)$.

El análisis de la superficie de lagunas como indicador de la ocurrencia de ciclos secos o húmedos mostró un comportamiento similar entre las lagunas de Mar Chiquita (en el centro del país) y las lagunas de Carrilaufquen (en el norte de la Patagonia). Ambos casos mostraron un incremento de la superficie entre los años 2000 y 2003/2004, posiblemente asociado a un ciclo húmedo y una disminución a partir de 2005 posiblemente debido a un ciclo seco (Figura 3ayb). La laguna de Los Pozuelos (en el noroeste del país) mostró un comportamiento inverso: una superficie muy reducida en la primera mitad del período analizado (ciclo seco) y un incremento en la segunda mitad (ciclo húmedo) (Figura 3c).

\section{DISCUSIÓN}

El análisis de la tendencia temporal de NDVI-I, como un indicador para identificar áreas sometidas a procesos de degradación o recuperación/ regeneración de la vegetación, ha sido aplicado en varias regiones del mundo (Evans \& Geerken, 2004; Herrmann et al., 2005; Wessels et al., 2007; Baldi et al., 2008). En la Argentina, la tendencia de NDVI-I en el período 2000-2014 mostró un patrón con una marcada heterogeneidad espacial, lo que sugiere la existencia de diferentes procesos que actúan a escala regional. Las áreas con tendencia negativa más acentuadas se hallaron en el norte de la región patagónica y en la región del Chaco Seco, mientras que las áreas con tendencia positiva se concentraron en las regiones Altoandina, Puna y Monte de Sierras y Bolsones. A continuación se discuten las posibles causas que podrían estar asociadas al comportamiento de NDVI-I en estas tres regiones del país.

\section{Región norte de la Patagonia}

La región comprendida por las provincias de 


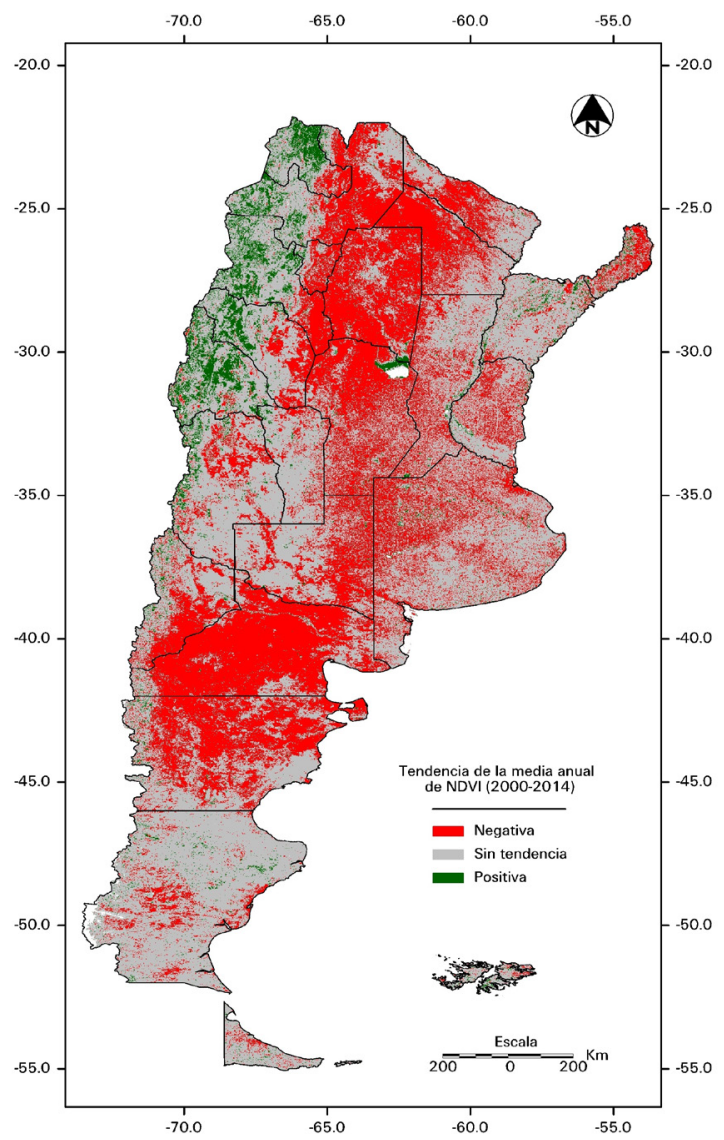

Figura 1. Tendencia de la integral anual del NDVI (NDVI-I) en el período 2000-2014 en la República Argentina.

Río Negro, Chubut y, en menor medida, Neuquén mostró una tendencia negativa en NDVI-I. Dentro de esta región, el área con una disminución más acentuada en este índice coincide con la región afectada por la deposición de cenizas del volcán Caulle-Puyehue (Figura 4), que entró en erupción el 4 de junio de 2011 y cubrió de cenizas la región con un espesor variable entre 30 y $0,2 \mathrm{~cm}$, de acuerdo a la distancia al volcán (Gaitán et al., 2012). Las cenizas depositadas sobre las hojas producen un efecto de "sombreado", reduciendo la cantidad de luz solar que las plantas pueden interceptar, lo que reduce la fotosíntesis y, en consecuencia, la productividad primaria de la vegetación (Oyarzabal et al., 2011). Irisarri et al. (2012) calcularon, mediante imágenes satelitales, una disminución de la productividad primaria de 40-50\% en el sudoeste de la provincia de Río Negro en los seis meses posteriores a la erupción. Además, la región norte de la Patagonia ha sido afectada por un ciclo seco, que se inició en 2005 y se intensificó

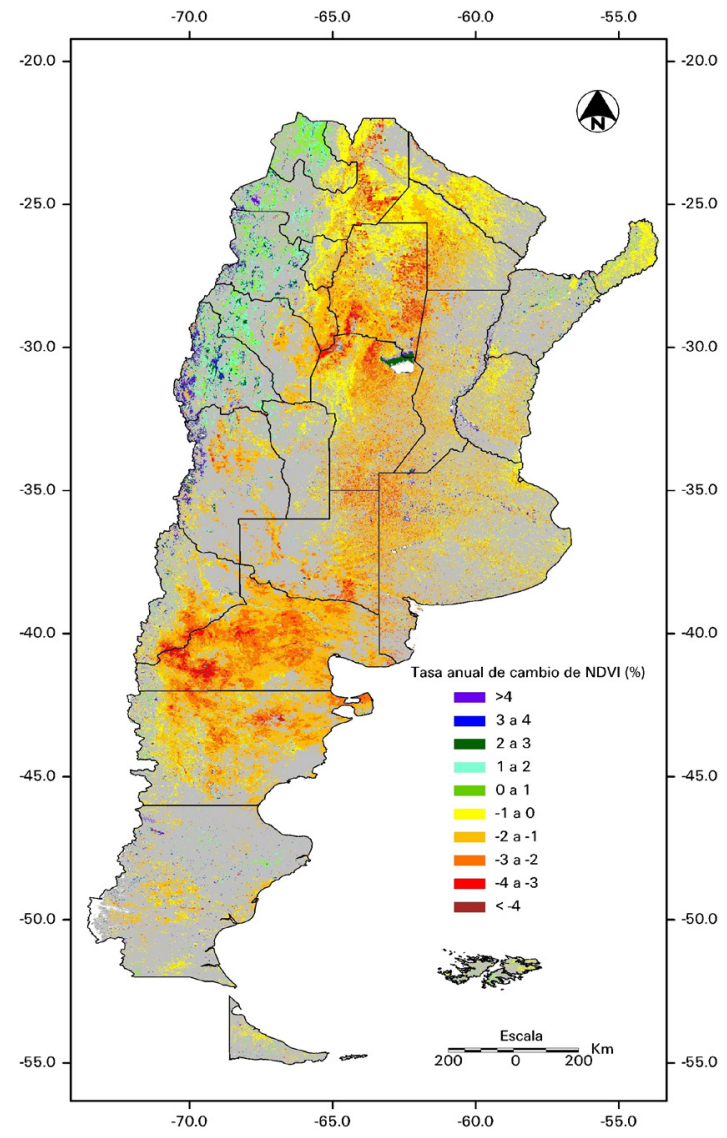

Figura 2. Tasa de aumento o disminución anual de NDVI-I (\%) en el período 2000-2014 en la República Argentina.

a partir del año 2007 (Villagra et al., 2011, 2012), asociado a la ocurrencia de años "Niña", que se evidencia en la fuerte disminución del tamaño de las lagunas de Carrilaufquen (Figura 3a). La combinación de cenizas volcánicas y sequía puede explicar la fuerte tendencia negativa de NDVI-I observada en esta región.

\section{Región del Chaco Seco}

En esta región los sectores con caídas más abruptas en NDVI-I presentan bordes geométricos (Figura 5), coincidiendo con áreas donde la vegetación natural ha sido reemplazada por cultivos anuales (REDAF, 2014). El proceso de cambio del uso del suelo, dominado por el incremento de la superficie agrícola a expensas de ecosistemas nativos, se ha intensificado a partir del año 2000, con el registro de las tasas de deforestación más altas de la historia en la Argentina (1,15\% anual) 

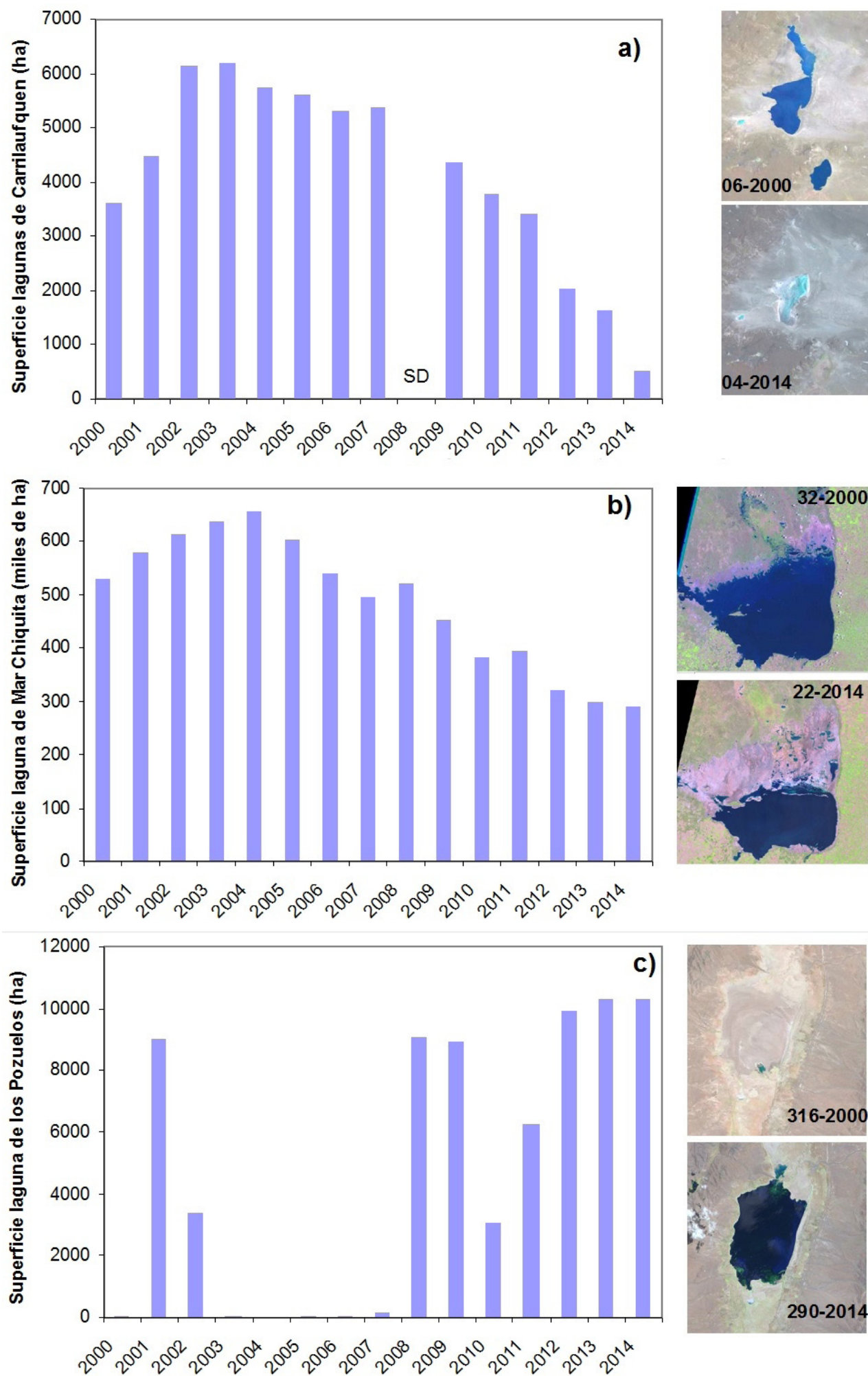

Figura 3. Evolución de la superficie de las lagunas de (a) Carrilaufquen Grande y Chica (Pcia. de Río Negro), (b) Mar Chiquita (Pcia. de Córdoba) y (c) de Los Pozuelos (Pcia. de Jujuy) en el período 2000-2014. Se muestran imágenes del sensor Landsat de cada laguna en el año 2000 y 2014, indicando el día juliano de cada imagen. 

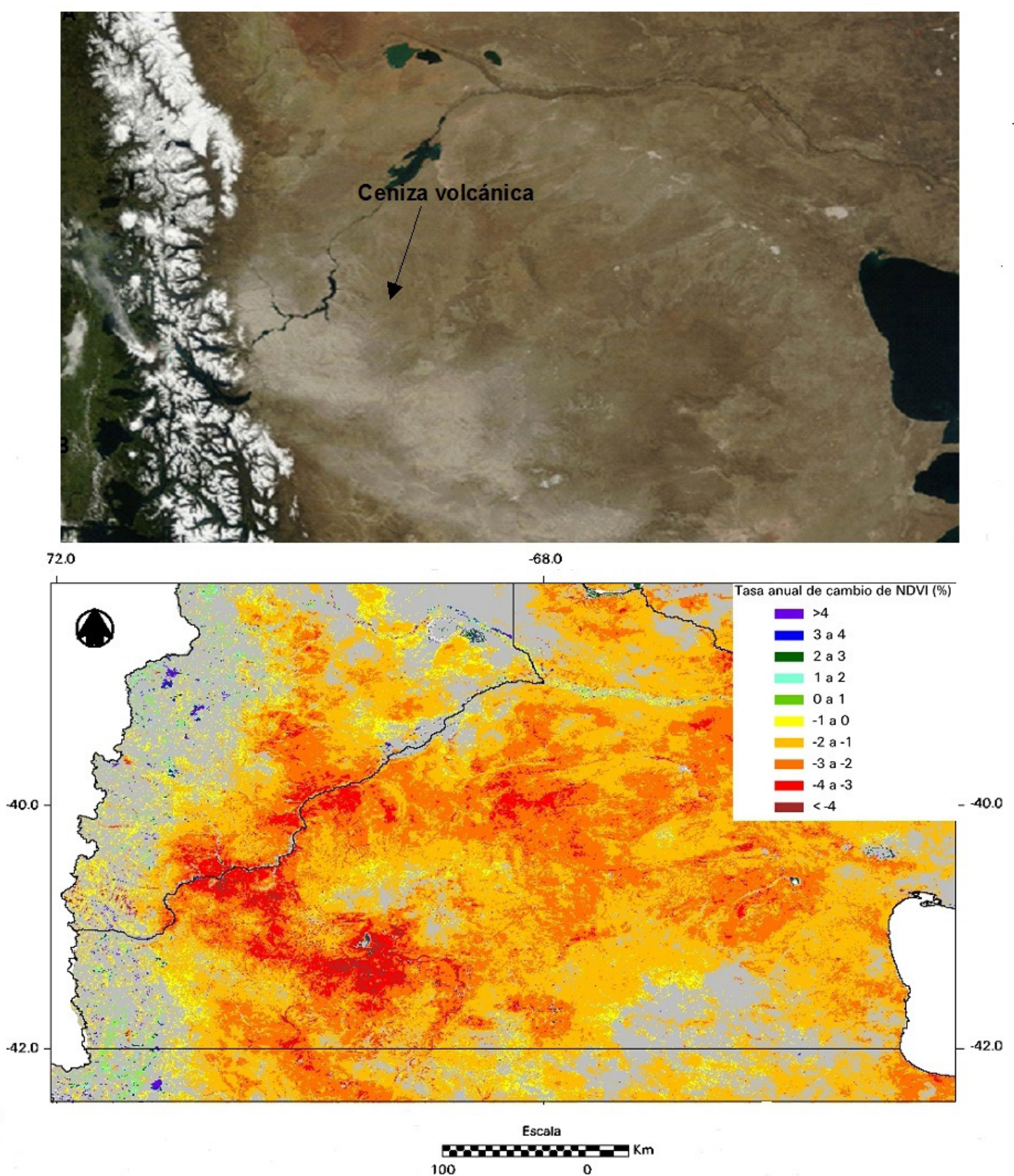

Figura 4. Correspondencia entre el área afectada por la deposición de cenizas del volcán Caulle-Puyehue ( $A$; visto en una imagen satelital MODIS) y el área que muestra caídas más acentuadas en NDVI-I en el norte de la Patagonia (B; sectores rojos en el mapa).

(Boletta et al., 2006; Gaspari \& Grau, 2009; Volante et al., 2012). El reemplazo de ecosistemas nativos por cultivos anuales produce cambios estructurales y funcionales que inciden en procesos como las ganancias de carbono y dinámica del agua, y conducen a la pérdida de biodiversidad y a la reducción de la capacidad de provisión de servi- cios ecosistémicos (Paruelo et al., 2005). Volante et al. (2012) observaron que el reemplazo de ecosistemas nativos por cultivos anuales produjo un incremento en la estacionalidad de la productividad de la vegetación, dado que los cultivos dejan el suelo desnudo durante un período prolongado del año. Entre otras consecuencias, esto produce 


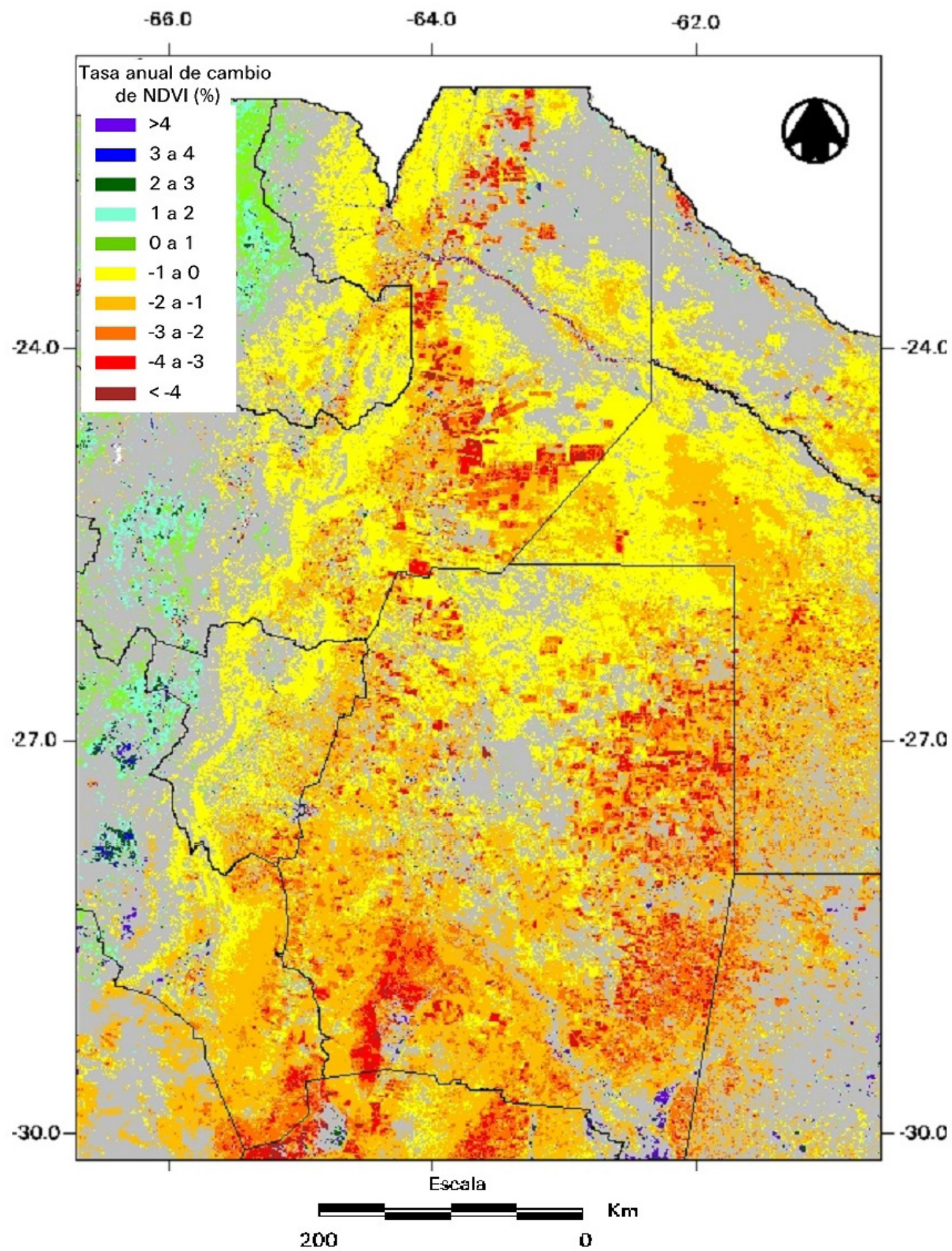

Figura 5. Tasa de aumento o disminución anual (\%) de NDVI-I en el período 2000-2014 en las provincias del centro-norte de la República Argentina. 
un aumento de las pérdidas de suelo por erosión. Dentro de las áreas desmontadas, el presente estudio discrimina zonas donde los cambios en NDVI-I fueron más o menos acentuados, lo cual puede estar relacionado con la magnitud de pérdida de servicios ecosistémicos. Además, la dominancia de años "Niña" en la segunda mitad del período analizado está relacionado con la ocurrencia de un ciclo seco en esta región, lo cual se evidencia en la progresiva disminución de la superficie de la laguna de Mar Chiquita a partir del año 2005 (Figura 3b). Zhao \& Running (2010) analizaron la tendencia de la productividad primaria, a nivel global, en el período 2000-2009, y hallaron, para esta región, resultados similares y los asociaron con la ocurrencia de años secos.

\section{Regiones Altoandina, Puna y Monte de Sierras y Bolsones}

Las áreas con tendencia positiva, que representan el 5\% del territorio nacional, se encuentran concentradas en estas regiones del país. Este comportamiento puede estar dado por la dominancia de años "Niña", en la primera mitad del período analizado, y años "Niño" en la segunda mitad. En estas regiones, a la inversa de lo observado en Patagonia y en el Chaco Seco, los años "Niña" están asociados a períodos secos y los años "Niño" a períodos húmedos, tal como se evidencia en la evolución de la superficie de la laguna de Los Pozuelos (Figura 3c).

\section{Ventajas y limitaciones de la metodología utilizada}

El uso de índices de vegetación derivados de sensores remotos tiene algunas ventajas sobre el uso de indicadores estructurales clásicos (como la riqueza de especies o la fisionomía de la vegetación): i) responden más rápido frente a perturbaciones de origen antrópico o natural (Milchunas \& Lauenroth, 1995) y ii) permiten monitorear grandes áreas bajo una aproximación común (Mildrexler et al., 2007), por lo que no es necesario definir protocolos de intra o extrapolación de observaciones puntuales (Paruelo, 2008). Así, el análisis de bases de datos de imágenes de satélite de largo plazo constituye una excelente herramienta para analizar los cambios funcionales de los ecosistemas (Alcaraz-Segura et al., 2008).

Una de las principales limitaciones de esta metodología como indicadora de la degradación de las tierras, es la dificultad de establecer relaciones causa-efecto en la tendencia de NDVI-I, dado que las variaciones en este índice pueden estar controladas por varios factores, y por lo tanto es difícil separar los efectos de ciclos climáticos de los efectos del uso antrópico (de Jong et al., 2011). Esto es especialmente difícil cuando no se cuentan con series de datos climáticas completas que permitan relacionar las variaciones de NDVI-I con las variaciones en el clima (principalmente en las precipitaciones), tal como fue el caso del presente estudio, en el cual no se contaron con mapas climáticos a escala nacional. Además, esta metodología se debería complementar con indicadores de procesos de degradación de tierras que pueden no ser bien captados por el NDVI tales como la contaminación de suelos y aguas y la pérdida de nutrientes y materia orgánica, principalmente en las regiones agrícolas.

El análisis de tendencia de NDVI-I puede indicar el efecto de procesos de largo plazo de degradación de las tierras debido al uso antrópico y también el efecto de eventos naturales de más corta duración (por ejemplo, ciclos secos o húmedos, deposición de cenizas volcánicas). Sería necesario distinguir entre ambos tipos de causas, para ello es necesario contar con datos auxiliares que permitan interpretar las variaciones de NDVI-I y analizar sus causas en cada región.

\section{CONCLUSIONES}

La metodología presentada, basada en el análisis de la tendencia temporal del índice NDVI derivado del sensor MODIS, permite observar patrones generales de tendencias positivas o negativas en la actividad de la vegetación (como un indicador del funcionamiento ecosistémico) y la tasa a la que ocurren estos procesos. Sin embargo, los cambios observados, que obedecen a causas naturales (por ejemplo, ciclos secos/húmedos, deposición de cenizas volcánicas) y a causas antrópicas (por ejemplo, desmontes para agricultura), deberían ser discriminados a partir de información complementaria. Esta metodología basada en índices de sensores remotos podría formar parte de un sistema de monitoreo de la degradación de las tierras a escala nacional, para lo cual debería ser validada con indicadores medidos a campo.

\section{AGRADECIMIENTOS}

Este trabajo fue financiado por el Observatorio Nacional de la Degradación de Tierras y Desertificación. 


\section{BIBLIOGRAFÍA}

Alcaraz-Segura, D.; G. Baldi, P. Durante y M.F. Garbulsky, 2008. Análisis de la dinámica temporal del NDVI en áreas protegidas: tres casos de estudio a distintas escalas espaciales, temporales y de gestión. Ecosistemas 17:108-117.

Baldi, G.; M.D. Nosetto, M.R. Aragón, F. Aversa, J.M. Paruelo and E.G. Jobbágy, 2008. Long-term sateIlite NDVI datasets: Evaluating their ability to detect ecosystem functional changes in South America. Sensors 8:5397-5425.

Boletta, P.E.; A.C. Ravelo, A.M. Planchuelo and M. Grilli, 2006. Assessing deforestation in the Argentine Chaco. Forest Ecology and Management 228:108-114

Chase, T.N.; R.A. Pielke, T.G.F. Kittel, R.R. Nemani and S.W. Running, 2000. Simulated impacts of historical land cover changes on global climate in northern winter. Climate Dynamics 16:93-105.

de Jong, R.; S. de Bruin, A. de Wit, M.E. Schaepman and D.L. Dent, 2011. Analysis of monotonic greening and browning trends from global NDVI time-series. Remote Sensing of Environment 115:692-702.

Evans, J. and R. Geerken, 2004. Discrimination between climate and human-indiced dryland degradation. Journal of Arid Environments 57:535-554.

Gaitán, J.J.; J.A. Ayesa, F. Umaña, F. Raffo y D.E. Bran, 2012. Cartografía del área afectada por cenizas Del volcán puyehue en Río Negro y Neuquén. XIX Congreso Argentino de la Ciencia del Suelo, Mar del Plata, 16 al 20 de abril de 2012

Gaitán, J.J.; D. Bran, G. Oliva, G. Ciari, V. Nakamatsu, J. Salomone, D. Ferrante, G. Buono, V. Massara, G. Humano, D. Celdrán, W. Opazo and F.T. Maestre, 2013. Evaluating the performance of multiple remote sensing indices to predict the spatial variability of ecosystem structure and functioning in Patagonian steppes. Ecological Indicators 34:181-191.

Gaspari, N.I. and H.R. Grau, 2009. Deforestation and fragmentation of Chaco dry forest in NW Argentina (19722007). Forest Ecology and Management 258:913-921.

Herrmann, S.M.; A. Anyamba and C.J. Tucker, 2005. Recent trends in vegetation dynamics in the African Sahel and their relationship to climate. Global Environmental Change 15:394-404.

Irisarri, J.G.; M. Oesterheld, J.M. Paruelo, P. Baldassini, D. Arocena y M. Oyarzabal, 2012. Impacto de la erupción del volcán Puyehue y el déficit de precipitaciones sobre la producción de materia seca del suroeste de Río Negro. LART - Laboratorio de Análisis Regional y Teledetección, IFEVA-FAUBA-CONICET, 13 pp.

Justice, C.; J. Townshend, E. Vermote, E. Masuoka, R. Wolfe, N. Saleous, D.P. Roy and J.T. Morisette, 2002.
An overview of MODIS Land data processing and product status. Remote Sensing of Environment 83:3-15.

McNaughton, S.J.; M. Oesterheld, D.A. Frank and K.J. Williams, 1989. Ecosystem-level patterns of primary productivity and herbivory in terrestrial habitats. Nature 341:142-144.

MEA (Millennium Ecosystem Assessment), 2005. Ecosystems and Human Well-being: Biodiversity Synthesis. World Resource Institute, Washington, DC, USA. <http://www.millenniumassessment.org/documents/ document.354.aspx.pdf > Consultada el 11/12/2015.

Milchunas, D.G. and W.K. Lauenroth, 1995. Inertia in plant community structure: State changes after cessation of nutrient enrichment stress. Ecological Applications 5:1195-2005.

Mildrexler, D.J.; M. Zhao, F.A. Heinsch and S.W. Running, 2007. A new satellite-based methodology for continental-scale disturbance detection. Ecological Applications 17:235-250.

Oyarzabal, M.; J.M. Paruelo, P. Baldassini y S. Aguiar, 2011. Impacto de la erupción del volcán Puyehue sobre la productividad forrajera de Chubut. LART - Laboratorio de Análisis Regional y Teledetección, IFEVAFAUBA-CONICET, 20 pp.

Paruelo, J.M, 2008. La caracterización funcional de ecosistemas mediante sensores remotos. Revista Ecosistemas 17:4-22.

Paruelo, J.M.; H.E. Epstein, W.K. Lauenroth and I.C. Burke, 1997. ANPP estimates from NDVI for the Central Grassland Region of the US. Ecology 78:953-958.

Paruelo, J.M.; J. Guerschman y S.R. Verón, 2005. Expansión agrícola y cambios en el uso del suelo. Ciencia Hoy 15:14-23.

Prince, S.D., 1991. Satellite remote sensing of primary production: comparison of results for Sahelian grasslands 1981-1988. International Journal of Remote Sensing 12:1301-1311

REDAF (Red Agroforestal Chaco Argentina), 2014. Monitoreo de desmontes en el Chaco Seco. <http://monitoreodesmonte.com.ar> Consultada el $11 / 12 / 2015$.

Reynolds, J.F. and D.M. Stafford Smith, 2002. Global Desertification: Do humans cause deserts? Vol. 88. Dahlem University Press, Berlin, 437 pp.

SADyS (Secretaría de Ambiente y Desarrollo Sustentable), 2001. Indicadores de impacto socioeconómico de Desertificación y Degradación de Tierras. Proyecto GER/01/S09 Avance de la Frontera Agropecuaria.

Steltzer, H. and J.M. Welker, 2006. Modeling the effect of photosynthetic vegetation properties on the NDVI-LAI relationship. Ecology 87:2765-2772

Tucker, C.J., 1979. Red and photographic infrared linear combinations for monitoring vegetation. Remote Sen- 
sing of Environment 8:127-150.

Tucker, C.J.; C.L. Vanpraet, E. Boerwinkel and A. Gaston, 1983. Satellite remote-sensing of total dry-matter production in the Senegalese Sahel. Remote Sensing of Environment 13:461-474.

Tucker, C.J.; C.L. Vanpreat, M.J. Sharman and G. Van Ittersum, 1985. Satellite remote sensing of total herbaceous biomass production in the Senegalese Sahel: 1980-1984. International Journal of Remote Sensing 7:233-249.

UNCCD., 1994. United Nations Convention to Combat Desertification, Intergovernmental Negotiating Committee For a Convention to Combat Desertification, Elaboration of an International Convention to Combat Desertification in Countries Experiencing Serious Drought and/or Desertification, Particularly in Africa. U.N. Doc. A/AC.241/27, 33 I.L.M. 1328. United Nations, New York, USA.

Villagra, S.; M.H. Easdale, L. Villar y C. Giraudo, 2011. Estado de situación y propuesta de acción para el área afectada por la sequía en la Provincia de Río Negro. INTA EEA Bariloche, 14 pp. <http://sipan.inta.gob.ar/ bibliografia/pdf/22.PDF> Consultada el 10/10/2014.
Villagra, S.; J.J. Gaitán y M.H. Easdale. 2012. Informe sobre la situación de la producción ganadera afectada por la sequía en la provincia de Neuquén. INTA EEA Bariloche. 11 páginas. <http://sipan.inta.gob.ar/ bibliografia/pdf/24.PDF> Consultada el 10/10/2014.

Volante, J.N.; D. Alcaraz-Segura, M.J. Mosciaro, E.F. Viglizzo and J.M. Paruelo, 2012. Ecosystem functional changes associated with land clearing in NW Argentina. Agriculture, Ecosystems and Environment 154:1222.

Wessels, K.J.; S.D. Prince, J. Malherbe, J. Small, P.E. Frost and D. Van Zyl, 2007. Can human-induced land degradation be distinguished from the effects of rainfall variability? A case study in South Africa. Journal of Arid Environments 68:271-297.

Wolter, K. and M.S. Timlin, 1993. Monitoring ENSO in COADS with a seasonally adjusted principal component index. Proc. of the 17th Climate Diagnostics Workshop, Norman, OK, NOAA/NMC/CAC, NSSL, Oklahoma Clim. Survey, CIMMS and the School of Meteor., Univ. of Oklahoma, pp. 52-57.

Zhao, M. and S.W. Running, 2010. Drought-induced reduction in global terrestrial net primary production from 2000 through 2009. Science 32:940-943. 